\title{
Prestations obligatoires de l'AOS et garanties de prise en charge: (auc)une contradiction?
}

Les garanties de prise en charge des coûts sont synonymes de rémunération pour les hôpitaux et de remboursement pour les patients. En revanche, elles provoquent une augmentation des charges administratives chez les médecins. Alors que des garanties de prise en charge tardives ou pour une courte durée suscitent souvent l'incompréhension des médecins et des patients, une rencontre de la $\mathrm{FMH}$, initiée par la commission spécialisée SwissDRG et le groupe de suivi «ST Reha» s'est penchée sur les possibilités de simplifier le processus de demande.

Petra Ingenpass ${ }^{a}$

Gabriela Lang ${ }^{b}$

a Dr en médecine, cheffe suppléante de la division Tarifs et économie de la santé pour les médecins hospitaliers

b Avocate, cheffe suppléante de la division Service juridique de la FMH
Correspondance: $\mathrm{FMH}$

Frohburgstrasse 15

CH-4600 Olten

Tél. 0313591111

Fax 0313591112

tarife.spital[at]fmh.ch

\section{Faut-il une garantie de prise en charge pour les prestations de l'assurance obligatoire de soins (AOS)?}

En principe, la loi sur l'assurance-maladie (LAMal) présume que les prestations médicales sont efficaces, adéquates et économiques (critères EAE) et, donc, qu'elles sont prises en charge par les assureurs-maladie. Pourtant, certaines dispositions contractuelles et juridiques exigent une garantie de prise en charge par l'assureur avant une hospitalisation prévue ou une réadaptation. Tandis que cette garantie offre aux hôpitaux l'assurance d'être remboursés, elle représente, pour les assureurs, chargés de contrôler les critères EAE, en particulier la nécessité d'une hospitalisation ou d'une réadaptation [1]. Lors d'un traitement extra-cantonal, les garanties de prise en charge indiquent aux patients les éventuels coûts qui ne seront pas couverts. Certaines prestations ambulatoires comme la poursuite de la psychothérapie après quarante séances ou des traitements oncologiques «off-label use» exigent également des garanties de prise en charge [2]. Conformément à l'ordonnance sur les prestations dans l'assurance obligatoire des soins en cas de maladie (OPAS), les coûts d'une réadaptation sont pris en charge «seulement si l'assureur a donné préalablement une garantie spéciale et avec l'autorisation expresse du médecin-conseil» [3].

La charge administrative, parfois considérable, liée à la demande de prise en charge ainsi que les délais des assureurs dans l'octroi de cette garantie posent problème aux médecins. Même dans les cas d'une réadaptation hospitalière clairement indiquée, il est fréquent qu'une garantie de prise en charge ne soit octroyée que pour une durée limitée impliquant par conséquent des demandes de prolongation en cours de traitement - ce qui entraîne une insécurité pour le patient comme pour le médecin. Par ailleurs, les délais des assureurs pour traiter les dossiers, qui peuvent atteindre jusqu'à cinq jours ou plus, sont jugés beaucoup trop longs par les médecins et les patients.

\section{Bases légales de la garantie de prise en charge des prestations médicales}

La loi sur l'assurance invalidité indique que tout traitement médical est soumis à une garantie de prise en charge préalable [4]; l'assurance-accidents, en revanche, laisse aux partenaires tarifaires - à défaut de dispositions légales ou réglementaires - le soin de clarifier la prise en charge.

La loi sur l'assurance-maladie prévoit la prise en charge, par les assureurs, des prestations conformes aux critères EAE. Le Conseil fédéral peut cependant désigner des prestations médicales qui ne sont pas soumises à une garantie de prise en charge, ou uniquement à certaines conditions [5]. Afin de garantir la qualité et l'adéquation des prestations médicales, il peut prévoir que certaines mesures diagnostiques ou thérapeutiques particulièrement coûteuses nécessitent l'accord préalable du médecin-conseil ou qu'elles ne soient pratiquées que par des fournisseurs de prestations qualifiés en la matière [6]. C'est pourquoi le Conseil fédéral et plus exactement le DFI a désigné dans l'OPAS (Annexe 1) les prestations médicales qui sont prises en charge par l'AOS, celles qui ne le sont pas ou qu'à certaines conditions. Cette liste n'est pas exhaustive. Mais pour certaines prestations de réadaptation notamment, la barre fixée est particulièrement haute.

\section{Octroi plus rapide, un point essentiel pour le patient}

L'enquête menée auprès des médecins par l'institut gfs.bern à la demande de la FMH a révélé que les assureurs retardaient souvent l'octroi de la garantie de prise en charge [7], dont le délai n'est réglementé ni dans la loi ni dans les ordonnances [8]. Les conventions tarifaires mentionnent des règles qui varient de 


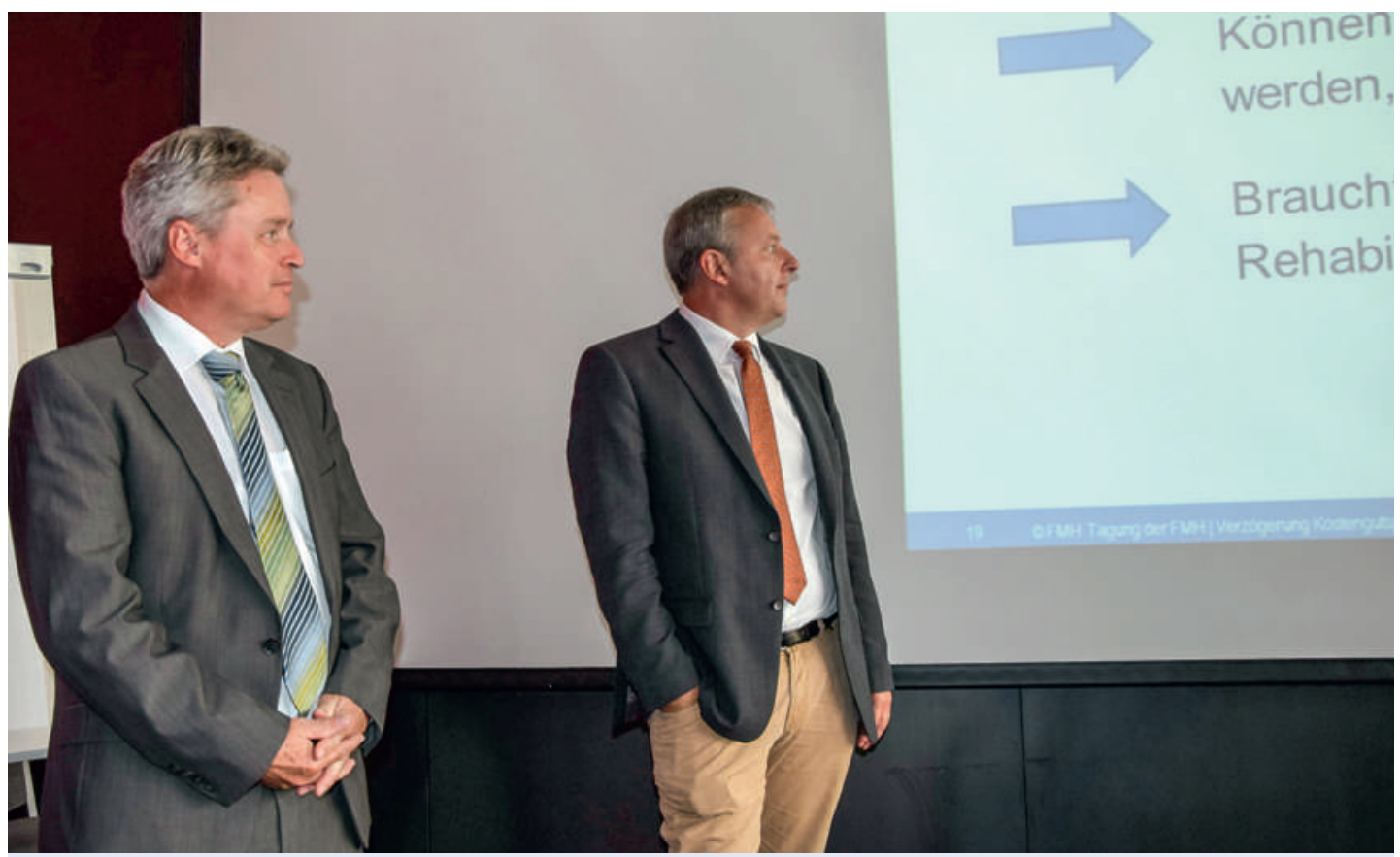

Stefan Wildi (à droite) et Beat Schärer répondent aux questions sur les garanties de prise en charge tacites.

«aussi vite que possible» à «en l'espace de cinq jours». Conformément aux droits fondamentaux de procédure, «toute personne a droit, dans une procédure judiciaire ou administrative, à ce que sa cause soit traitée équitablement et jugée dans un délai raisonnable» [9]. Les assureurs sont également soumis à ce droit fondamental. Avec l'assurance-maladie obligatoire, ils assument en effet une mission publique de la Confédération [10]. Mais qu'est-ce qu'un «délai raisonnable»? Selon la jurisprudence du Tribunal fédéral, il y a violation de ce principe fondamental lorsque le dossier est retardé à l'excès. La durée «raisonnable» doit être examinée au cas par cas: les circonstances concrètes, l'ampleur et la complexité du cas jouent ici un rôle déterminant [11]. Même si les

\section{Critères et définitions pour l'octroi}

\section{de garanties de prise en charge *}

- Recommandations concernant l'indication et l'application pratique de la réadaptation cardiaque

- Tableaux cliniques/indications de la réadaptation pulmonaire

- Réadaptation après une opération de la colonne vertébrale

- Remplacement de la hanche et du genou: réadaptation

- Médicaments «Off-label use»

- Critères pour la chirurgie élective de la main ambulatoire ou hospitalière

- Etc.

* Source: www.medecins-conseils.ch conventions tarifaires fixent un délai concret pour l'octroi d'une garantie de prise en charge, il est possible qu'un délai plus court ou plus long soit jugé raisonnable selon le cas d'espèce.

\section{Critères d'octroi d'une garantie de prise en charge}

Les sociétés de discipline médicale ont déjà rédigé souvent en collaboration avec les médecins-conseils et les médecins d'assurance - de nombreuses recommandations: par exemple les critères indiquant une réadaptation hospitalière ou ambulatoire après un remplacement de la hanche. Ces recommandations servent de base aux médecins-conseils pour décider de l'octroi d'une garantie de prise en charge. Leur prise de position à l'intention de l'assureur ne constitue toutefois qu'une recommandation sans caractère contraignant pour ce dernier [12].

\section{Garanties de prise en charge tacites - un projet-pilote à Zurich}

L'augmentation de la bureaucratie a poussé des médecins $\mathrm{du}$ «Cercle zurichois pour une gestion hospitalière progressiste» à formuler des propositions en vue de réduire la charge administrative - y compris celle liée aux garanties de prise en charge. Lors de la rencontre de la commission spécialisée SwissDRG et du groupe d'accompagnement ST Reha de la FMH en automne 2014, Stefan Wildi [13] et Beat Schärer [14] ont rendu compte de leurs premières expériences, qui font ressortir la forte charge administrative générée par les garanties de prise en charge pour les hôpitaux et les assureurs: les demandes de rensei- 
gnements internes à l'hôpital et de la part des assureurs, la préparation et l'échange de documents médicaux ainsi que l'absence d'interlocuteurs concrets à l'hôpital et au sein de l'assurance rendent les processus particulièrement complexes. D'autre part, Sanitas examine toutes les demandes de prise en charge au cas par cas alors que plus de 90\% d'entre elles sont approuvées.

En concertation avec le médecin-conseil de Sanitas, les hôpitaux Limmattal, Waid et Triemli ont établi une liste de maladies qui nécessitent dans tous les cas une réadaptation hospitalière, et convenu pour ces indications d'une «garantie de prise en charge tacite». Néanmoins, seul un petit nombre des cas répertoriés dans ces trois établissements correspondait exactement aux critères définis. Il n'a donc pas encore été possible de démontrer une simplification par rapport à la procédure habituelle; cela s'explique entre autres par le fait que les cliniques de réadaptation ont continué d'exiger des assureurs une confirmation de prise en charge.

Malgré l'absence d'amélioration fondamentale, ce projet-pilote a permis de prendre connaissance des processus de travail réciproques ainsi que des interlocuteurs personnels, et d'instaurer ainsi un meilleur climat de confiance entre les partenaires. Cette collaboration améliorée se reflète d'ores et déjà dans la diminution des demandes de renseignements. Il est désormais prévu d'étendre la liste des indications pour des garanties de prise en charge tacites et d'élargir le projet à des assureurs et à des cliniques de réadaptation supplémentaires.

\section{Garantie de prise en charge - solutions envisageables du point de vue des médecins}

Le corps médical juge problématique la réglementation inscrite dans l'OPAS selon laquelle chaque réadaptation hospitalière exige une garantie de prise en charge préalable avec l'accord exprès du médecin-conseil ainsi que sa mise en ouvre par les assureurs. La modification de cette formulation dans le cadre d'une révision de l'OPAS permettrait de résoudre le problème. Cette voie s'avère toutefois fastidieuse et n'a pas été retenue par les participants à la rencontre. En revanche, les sociétés de discipline médicale et les médecins-conseils doivent élaborer ensemble des directives détaillées et contraignantes qui définiront par exemple les indications et la durée minimale des prestations médicales. Ainsi, les garanties de prise en charge pourraient même devenir superflues. Sanitas considère, elle aussi, que de telles extensions et précisions de la part des sociétés de discipline médicale s'avéreraient précieuses et très utiles pour les médecins-conseils.

\section{Références}

1 La prise en charge par l'AOS d'un traitement hospitalier ou d'une réadaptation hospitalière est conditionnée par la nécessité d'hospitalisation. Selon le Tribunal fédéral, la nécessité de séjour hospitalier est avérée lorsqu'une maladie nécessite un traitement aigu ou une réadaptation hospitalière, mais également lorsque les mesures médicales nécessaires ne peuvent être fournies de manière adéquate ou avec succès qu'en milieu hospitalier après épuisement des possibilités ambulatoires. Hormis l'état de santé, la nécessité d'un séjour hospitalier peut également être déterminée par les conditions de vie personnelles du patient (ATF 126 V 323 cons. 2b)

2 Cf. art. 3b de l'ordonnance sur les prestations de l'assurance des soins (OPAS) et art. 71a f. de l'ordonnance sur l'assurance-maladie (OAMal).

3 Annexe 1 de l'OPAS, chapitre 11, Réadaptation.

4 Art. 8 al. 3 de la loi fédérale sur l'assurance-invalidité (LAI), en lien avec l'art. 78 al. 1 de l'ordonnance concernant l'assurance-invalidité.

5 Art. 33 al. 1 LAMal.

6 Art. 58 al. 3 LAMal.

7 Evolution des conditions-cadres pour les médecins sous www.fmh.ch $\rightarrow$ Tarifs hospitaliers $\rightarrow$ Recherche concomitante

8 Exception: pour la poursuite d'une psychothérapie médicale après 40 séances, la garantie de prise en charge doit être octroyée dans les 15 jours (art. 3b al. 4 OPAS).

9 Art. 29 al. 1 Consitution fédérale (Cst.); ATF 131 V 407 cons. 1.1.

10 Arrêt 1C_372/2014 du 4.9.2014 cons. 6 .

11 Arrêt 1B_549/2012 du 12.11.2012 cons. 2.3.

12 Gebhard Eugster, Jurisprudence du Tribunal fédéral sur la LAMal, Cm 4 de l'art. 57, Schulthess Zurich 2010.

13 Dr Stefan Wildi, privat-docent, médecin-chef de la clinique de chirurgie, Stadtspital Waid, Zurich.

14 Beat Schärer, responsable de la Gestion des prestations et membre de la Direction, Sanitas.

\section{Sujets d'actualité du forum \\ 000}

Venez débattre avec nous! Dans la rubrique forum, nous présentons régulièrement des sujets d'actualité politique, économique et scientifique ayant trait au système de santé. Donnez votre avis ou commentez les affirmations de vos confrères. Pour accéder au forum: www.bullmed.ch/forum/ 